\title{
Capsaicin Ameliorates Cisplatin-Induced Renal Injury through Induction of Heme Oxygenase-1
}

\author{
Sung-Hyun Jung ${ }^{1,2,3}$, Hyung-Jin Kim ${ }^{1,3}$, Gi-Su Oh ${ }^{1}$, AiHua Shen ${ }^{1,2}$, Subin Lee ${ }^{1,2}$, Seong-Kyu Choe ${ }^{1}$, \\ Raekil Park ${ }^{1,2}$, and Hong-Seob So ${ }^{1,2, *}$
}

\begin{abstract}
Cisplatin is one of the most potent chemotherapy agents. However, its use is limited due to its toxicity in normal tissues, including the kidney and ear. In particular, nephrotoxicity induced by cisplatin is closely associated with oxidative stress and inflammation. Heme oxygenase-1 (HO-1), the rate-limiting enzyme in the heme metabolism, has been implicated in a various cellular processes, such as inflammatory injury and anti-oxidant/oxidant homeostasis. Capsaicin is reported to have therapeutic potential in cisplatin-induced renal failures. However, the mechanisms underlying its protective effects on cisplatin-induced nephrotoxicity remain largely unknown. Herein, we demonstrated that administration of capsaicin ameliorates cisplatininduced renal dysfunction by assessing the levels of serum creatinine and blood urea nitrogen (BUN) as well as tissue histology. In addition, capsaicin treatment attenuates the expression of inflammatory mediators and oxidative stress markers for renal damage. We also found that capsaicin induces HO-1 expression in kidney tissues and HK-2 cells. Notably, the protective effects of capsaicin were completely abrogated by treatment with either the $\mathrm{HO}$ inhibitor ZnPP IX or HO-1 knockdown in HK-2 cells. These results suggest that capsaicin has protective effects against cisplatin-induced renal dysfunction through induction of HO-1 as well as inhibition oxidative stress and inflammation.
\end{abstract}

\section{INTRODUCTION}

cis-Diamminedichloroplatinum II (cisplatin) is a widely used chemotherapeutic agent to treat solid tumors in the testicles, ovaries, head and neck, bladder, lung, and uterus (Arany and Safirstein, 2003; Cohen and Lippard, 2001; Pabla and Dong,

${ }^{1}$ Center for Metabolic Function Regulation, Department of Microbiology, Wonkwang University School of Medicine, Iksan 570-749, Korea, ${ }^{2}$ Brain Korea 21 Plus Program and Department of Smart Life-Care Convergence, Wonkwang University, Graduate School, Iksan 570-749, Korea, ${ }^{3}$ These authors contributed equally to this work.

*Correspondence: jeanso@wku.ac.kr

Received 1 November, 2013; revised 4 February, 2014; accepted 13 February, 2014; published online 14 March, 2014

Keywords: capsaicin, cisplatin, $\mathrm{HO}-1$, nephrotoxicity
2008; Siddik, 2003; Wang and Lippard, 2005). However, significant nephrotoxicity has been reported with approximately $30 \%$ of the patients experiencing a significant reduction in renal function during cisplatin chemotherapy, and thus limits its use (Kuriakose and Kurup, 2008; Luke et al., 1992). Although the exact mechanisms of cisplatin-induced nephrotoxicity have still not been fully elucidated, a positive feedback loop between inflame-matory cytokines and oxidative stress causing tubular toxicity and vascular injury has been suggested to be responsible for cisplatin-induced renal failure (Kuhad et al., 2007; Maheshwari et al., 2013; Mitazaki et al., 2013; Sahu et al., 2013). Therefore, it is suggested that modulation of the inflammatory response and oxidative stress can be potentially useful to attenuate cisplatin-induced nephrotoxicity.

Heme oxygenase $(\mathrm{HO})$, the rate-limiting enzyme in the catabolism of heme, converts heme into biliverdin, carbon monoxide, and iron. Induction of $\mathrm{HO}-1$ was reportedly associated with most protective mechanisms activated by various oxidative insults such as heme, hyperoxia, hypoxia, and electrophiles, and thus it is thought to play a key role in maintaining the oxidant/anti-oxidant balance (Hartsfield et al., 1999; Lee et al., 2000; Maines, 1988; Owuor and Kong, 2002). In particular, chemical inhibition of $\mathrm{HO}$ enzyme activity in rats or genetic knockout of HO-1 in mice results in much worsened kidney dysfunction and tubular injury in response to cisplatin treatment, indicating a protective role for $\mathrm{HO}-1$ expression in cisplatininduced renal tubular cell death (Agarwal et al., 1995; Shiraishi et al., 2000).

Capsaicin (trans-8-methyl- $N$-vanillyl-6-nonenamide), the active ingredient of chili peppers, was originally used in the study of pain sensations through the stimulation of its receptor (Caterina et al., 1997; Szallasi and Blumberg, 1999). More recently, capsaicin has been reported to have anti-mutagenic and anticarcinogenic properties against human leukemic and hepatic carcinoma cells (Huang et al., 2009; Zhang et al., 2003). Indeed, capsaicin potently inhibited lipid peroxidation and ROS generation in rat peritoneal macrophages and the inflammatory response in the sepsis model (Joe and Lokesh, 1994; Kogure et al., 2002; Okada and Okajima, 2001). Moreover, administration of capsaicin was shown to exert protective effect against cisplatin-induced nephrotoxicity in rats (Shimeda et al., 2005). However, the mechanism responsible for the potent inhibitory effects of capsaicin on cisplatin-induced nephrotoxicity is still unclear. In the present study, we investigated the molecular mechanism of capsaicin and found that the protective effect of 
capsaicin on cisplatin-induced nephrotoxicity is mediated by HO-1 induction which inhibits oxidative and inflammatory events.

\section{MATERIALS AND METHODS}

\section{Animals and drug treatments}

Male C57BL/6 mice (7 week old, weighing 20-22 g) were purchased from Orient Bio (Korea) and maintained according to a standard animal protocol approved by the Animal Care and Use Committee at Wonkwang University. The dose of cisplatin and the time of treatment were selected based on the previous study (Shimeda et al., 2005). Capsaicin (2.5, 5, and $10 \mathrm{mg} / \mathrm{kg}$ body weight) was administered orally once a day for 5 consecutive days. Cisplatin (5 mg/kg body weight) was injected once, $12 \mathrm{~h}$ after capsaicin administration for 4 consecutive days. The mice were sacrificed the day after the final cisplatin injection.

\section{Assays for renal functional markers and pro-inflammatory cytokines}

For renal function analysis, serum was isolated and stored at $-80^{\circ} \mathrm{C}$ until use. Serum creatinine and blood urea nitrogen (BUN) levels were measured using an assay kit according to the manufacturer's instructions (BioVision, USA). The proinflammatory cytokines TNF- $\alpha, \mathrm{IL}-1 \beta$, and IL- 6 in each serum were quantified by ELISA (Quantikine Kit; R\&D Systems, USA) according to the manufacturer's instructions.

Histological evaluation and Immunohistochemical analysis Kidney tissues were fixed in $4 \%$ formaldehyde and embedded in paraffin wax. The $5-\mu \mathrm{m}$-thick sections were deparaffinized in xylene and rehydrated through graded concentrations of ethanol. For histopathological analysis, hematoxylin and eosin $Y$ (H\&E) staining was performed using standard protocols. Immunohistochemistry (IHC) was performed using an IHC kit (DAKO LSAB Universal K680, USA). Briefly, kidney sections were incubated in a blocking solution with $5 \%$ bovine serum albumin followed by a solution containing primary antibody (1:200 dilution) with $1 \%$ bovine serum albumin for $1 \mathrm{~h}$. After repeated washes with PBS, each section was incubated with biotinylated secondary antibody for $1 \mathrm{~h}$ and then covered for $15 \mathrm{~min}$ with streptavidin-peroxidase. Finally, after repeated washes with PBS, each section was stained in a freshly -prepared substrate solution (3 mg of 3-amino-9-ethylcarbazole in $10 \mathrm{ml}$ of sodium acetate buffer, $\mathrm{pH} 4.9,500 \mu$ l of dimethylformamide, and $0.03 \%$ hydrogen peroxide) for $10 \mathrm{~min}$. The nuclei of immunostained cells were counterstained with Mayer's hematoxylin (SigmaAldrich, USA) and then images were collected using a light microscope (IX71, Olympus, Japan).

\section{Cell culture and viability}

HK-2 cells (ATCC, USA), an immortalized human proximal tubular epithelial cell line, were maintained in Dulbecco's modified eagle's medium-F12 (Invitrogen, USA) supplemented with $10 \%$ fetal bovine serum (FBS) and antibiotics $(100 \mathrm{U} / \mathrm{ml}$ penicillin $\mathrm{G}, 100 \mu \mathrm{g} / \mathrm{ml}$ streptomycin, and $0.25 \mu \mathrm{g} / \mathrm{ml}$ amphotericin $\mathrm{B}$ ). Cells $\left(3 \times 10^{4}\right.$ cells per well of a 24 -well plate) were plated for $16 \mathrm{~h}$ and incubated with cisplatin $(30 \mu \mathrm{M})$ dissolved in water in the presence of varying concentrations of capsaicin in ethanol for $24 \mathrm{~h}$. To determine the cell viability, MTT $(0.25 \mathrm{mg})$ was added to $1 \mathrm{ml}$ of the cell suspension for $2 \mathrm{~h}$. After washing the cells three times with PBS, $\mathrm{pH} 7.4$, the insoluble formazan product was dissolved in DMSO. Optical density (O.D.) was measured using a microplate reader (Titertek Multiskan; Flow Laboratories, USA) at $590 \mathrm{~nm}$. The O.D. in the control cells was declared as $100 \%$ of viability.

\section{RNA interference}

Predesigned siRNA to human $\mathrm{HO}-1$ and control scrambled siRNA were purchased from Santa Cruz Biotechnology (USA). HK-2 cells were transiently transfected with $100 \mathrm{nM}$ siRNA constructs using X-tremeGENE siRNA transfection reagent (Roche Applied Science, Germany), according to the manufacturer's protocol. The knockdown of protein expression was confirmed by immunoblot analysis.

\section{Immunoblot analysis}

To determine the expression levels of target proteins, Western blots were performed. Briefly, kidney tissues and HK-2 cells were homogenized in lysis buffer $(10 \mathrm{mM}$ Tris- $\mathrm{HCl} \mathrm{pH}$ 7.6, 150 $\mathrm{mM} \mathrm{NaCl}, 1 \%$ Triton $\mathrm{X}-100,1 \%$ sodium deoxycholate, $1 \mathrm{mM}$ EDTA, $50 \mathrm{mM} \beta$-glycerophosphate, $1 \mathrm{mM}$ DTT, $1 \mathrm{mM} \mathrm{NaF}, 1$ $\mathrm{mM} \mathrm{Na}_{3} \mathrm{VO}_{4}, 1 \mathrm{mM} \mathrm{PMSF}$, and $1 \mathrm{X}$ protease inhibitor cocktail). The samples from kidney or HK-2 cells were incubated with anti-TLR4 (Santa Cruz Biotechnology Inc., USA) or anti-HO-1 antibody (Stressgen, Canada). Signals were visualized by a chemiluminescent solution according to the manufacturer's instructions (Supersignal Pico Substrate; Thermo Scientific, Pierce Chemical). The membranes were reprobed with anti-actin antibody (Santa Cruz Biotechnology Inc.) as a loading control. Signaling intensities were quantified using the ImageJ program.

Real-time PCR analysis

Total RNA was isolated from cells using TRIzol (Invitrogen, USA) according to the manufacturer's protocol. Three micrograms of RNA was converted to cDNA using the First Strand cDNA Synthesis Superscript kit (Invitrogen) according to the manufacturer's protocol. Quantitative real-time PCR was performed with 10 pmol of primers for $\mathrm{HO}-1$ and GAPDH using SYBR Green Mastermix (Invitrogen). Reactions were performed in triplicate and specificity was monitored using melting curve analysis after cycling. Primers used were as follows: mouse HO-1, 5'-GGT GAT GGC TTC CTT GTA CC-3' and 5'AGT GAG GCC CAT ACC AGA AG-3'; human HO-1, 5'-CAT GAC ACC AAG GAC CAG AG-3' and 5'-AGT GTA AGG ACC CAT CGG AG-3'; mouse NQO1, 5'-AGC CCA GAT ATT GTG GCC G-3' and 5'-CGT TTC AGA ATG GTC GGC AC-3'; mouse GCLC, 5'-CTG CAC ATC TAC CAC GCA GT-3' and 5'GTC TCA AGA ACA TCG CCT CC-3'; mouse GCLM, 5'-CGG GAA CCT GCT CAA CTG-3' and 5'-CCA AAA CAT CTG GAA ACT CCC-3'; mouse GSS, 5'-GAA GCA GCT CGA AGA ACT GG-3' and 5'-AGC ACT GGG TAC TGG TGA GG-3'; GAPDH, 5'-TCC CAC TCT TCC ACC TTC GA-3' and 5'-AGT TGG GAT AGG GCC TCT CTT G-3'. Relative mRNA expression was quantified using the $\triangle \Delta \mathrm{Ct}$ method and GAPDH was used as an internal control. Results were expressed as fold change.

\section{Determination of ROS production}

The intracellular ROS level was measured using a fluorescent dye $2^{\prime}, 7^{\prime}$ dichlorodihydrofluorescein diacetate (H2-DCFDA; Invitrogen). Cells were plated in 96-well culture plates overnight and treated with cisplatin in the presence or absence of capsaicin for $24 \mathrm{~h}$. After washing with PBS, serum-free DMEM containing $10 \mu \mathrm{M} \mathrm{H} 2$-DCFDA was added to each well and incubated at $37^{\circ} \mathrm{C}$ for $1 \mathrm{~h}$. ROS production was measured using a microplate reader equipped with a spectrofluorometer at an emission wavelength of $538 \mathrm{~nm}$ and extinction wavelength of $485 \mathrm{~nm}$. Relative ROS production was expressed as the change 

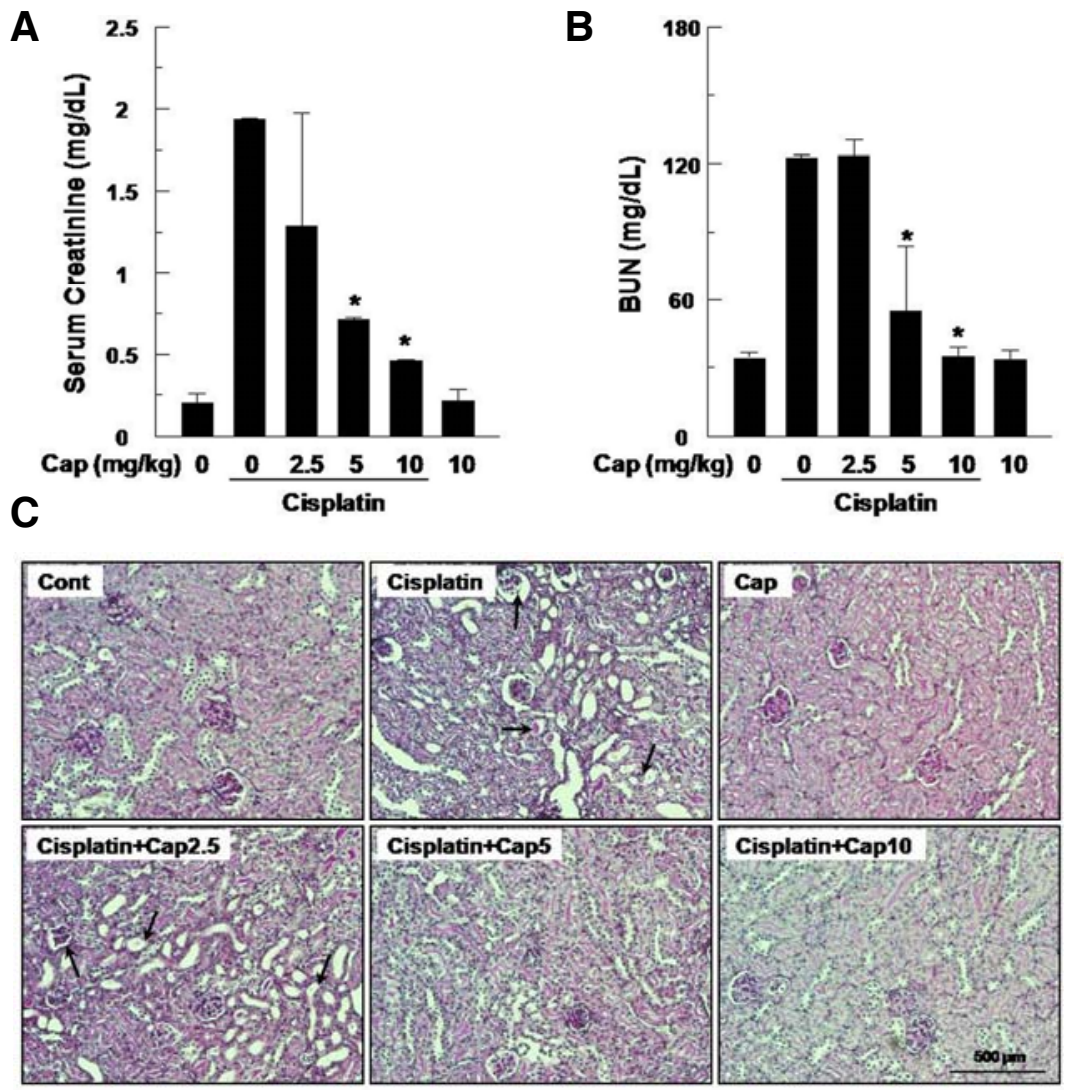

Fig. 1. Effect of capsaicin pretreatment on renal function and histology in cisplatin-induced kidney injury. Capsaicin was administered orally once a day for 5 consecutive days. Cisplatin was injected once at $12 \mathrm{~h}$ after capsaicin treatment for 4 consecutive days. The mice were sacrificed on day after the final cisplatin injection, and levels of serum creatinine $(A)$ and BUN (B) were analyzed by an assay kit according to the manufacturer's instructions (BioVision, USA). ${ }^{*} p<0.05$ by one-way ANOVA compared with the cisplatin group $(n=5)$. Kidney specimens were stained with H\&E (C). Damaged areas on tissue sections are marked with black arrows. in fluorescence of experimental groups compared with that of the appropriate controls.

\section{Statistical analysis}

Each experiment was performed at least three times, and al values represent the means \pm S.D. of triplicate analyses. Statistical multivariate analysis was performed by analysis of variance and Duncan tests, using the SPSS 11 (USA) statistical software. Two-way ANOVA and/or one-way ANOVA were used to determine the significance of the results. The statistical results were reviewed by a masters-level biostatistician. Values of $p<0.05$ were considered to be statistically significant.

\section{RESULTS}

\section{Capsaicin ameliorates cisplatin-induced kidney injury in} mice

To evaluate the effects of capsaicin on renal function, C57BL/6 mice were treated with capsaicin, cisplatin, or capsaicin plus cisplatin according to the experimental schedule described in the "Materials and Methods", and the levels of serum creatinine and BUN were measured. As shown in Figs. 1A and 1B, cisplatin alone caused a marked increase in serum creatinine and BUN as compared with control group. In contrast, capsaicin pretreatment significantly attenuated the cisplatin-mediated increase in a dose-dependent manner. Next, to examine the effect of capsaicin on the histological changes caused by cisplatin treatment, kidney specimens from experimental groups were stained with H\&E. As shown in Fig. $1 \mathrm{C}$, mice treated with cisplatin showed various degrees of tubular and glomerular damage, such as tubular dilation, vacuole formation, and necrosis. However, pretreatment with capsaicin dose-dependently prevented the renal damage, resulting in normal kidney morphology with slight changes in the glomeruli and minor edema of the tubular cells. These results suggest that capsaicin protects against cisplatin-induced renal injury.

\section{Capsaicin inhibits cisplatin-induced inflammatory responses in mice}

Because inflammatory mediators including pro-inflammatory cytokines, Toll-like receptor 4 (TLR4), and TLR4 ligands may exacerbate cisplatin-induced renal injury, we examined if capsaicin pretreatment altered expression of these cisplatininduced mediators. First, we assessed the serum levels of proinflammatory cytokines by ELISA. Cisplatin treatment resulted in a marked increase of pro-inflammatory cytokine secretion, specifically TNF- $\alpha$, IL-1 $\beta$, and IL- 6 . However, the increased secretion of these pro-inflammatory cytokines was significantly attenuated with capsaicin pretreatment in a dose-dependent manner (Figs. 2A-2C). We also examined the level of TLR4 and its ligands, HMGB1 and AGEs, in kidney tissue by Western blot and immunohistochemistry (IHC). The induction of TLR4 in cisplatin-treated kidney tissue was strongly attenuated by capsaicin pretreatment as shown by Western blot and IHC analysis (Figs. 2D and 2E). We also found that cisplatin treatment increased the expression of HMGB1 primarily in the glomeruli (Fig. 2F) and AGEs in the renal tubular cells (Fig. 2G), and these effects were almost completely abrogated by capsaicin pretreatment (Figs. 2F and $2 \mathrm{G}$ ). 

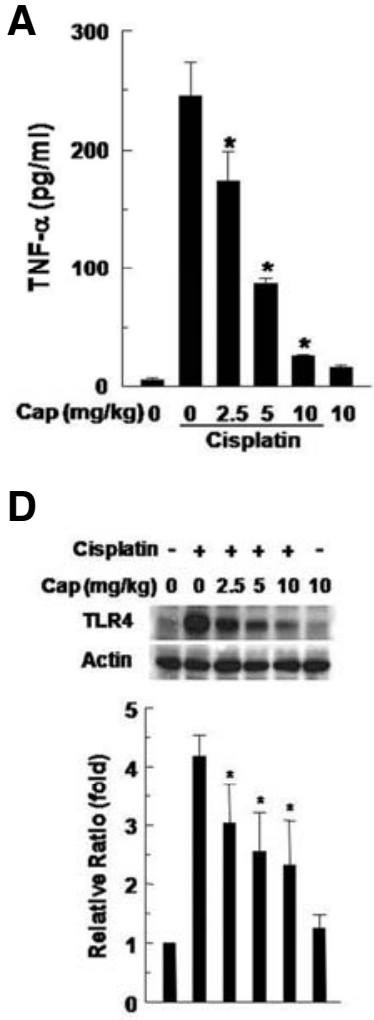
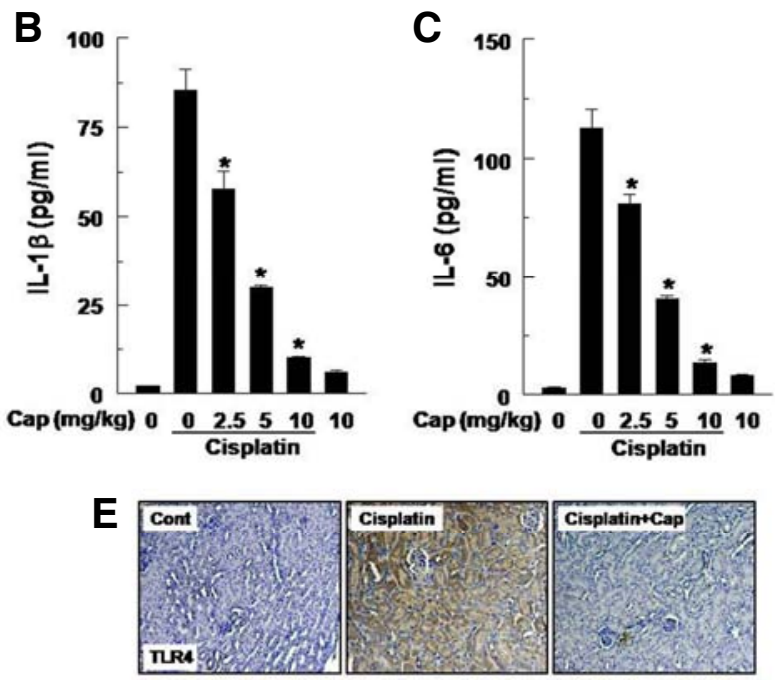

$\mathbf{F}$

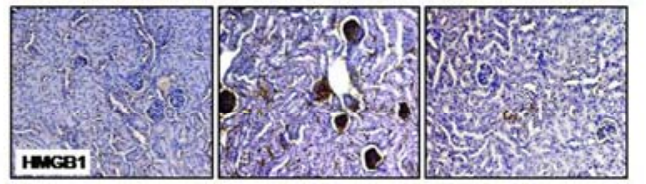

G

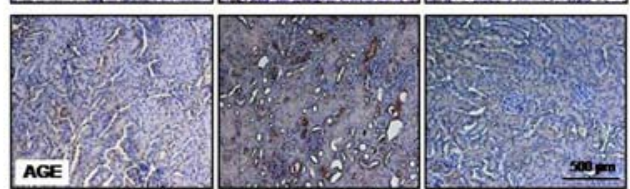

Fig. 2. Effect of capsaicin pretreatment on cisplatin-induced inflammatory mediators. TNF- $\alpha(A), I L-1 \beta(B)$, and IL-6 (C) were analyzed in serum by ELISA. Total kidney lysate was blotted and probed with an anti-TLR4 antibody (upper panel). (D) The blots were reprobed with an anti-actin antibody. Densitometric analyses are presented as the relative ratio of TLR4 to actin (lower panel). ${ }^{*} p<0.05$ by oneway ANOVA compared with the cisplatin group $(n=3)$. Kidney sections were immunohistochemically stained with TLR4 (E), HMGB1 (F), and AGEs (G) antibodies. Cont, PBS (phosphate-buffered saline)-treated group; Cisplatin, cisplatin only treated group; Cisplatin + Cap, cisplatin and $10 \mathrm{mg} /$ $\mathrm{kg}$ capsaicin-treated group.
A

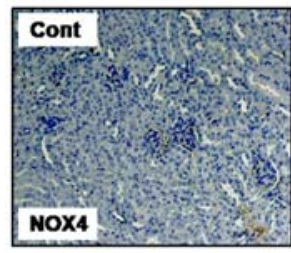

C

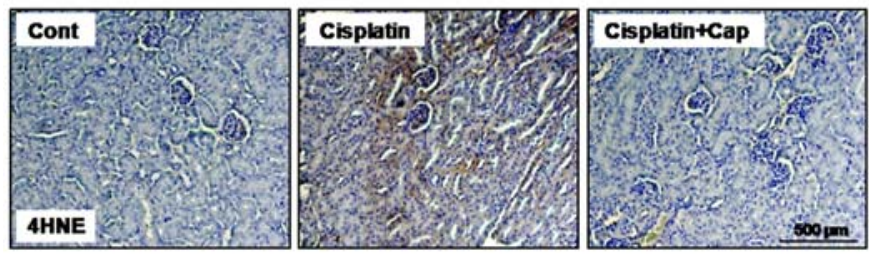

B
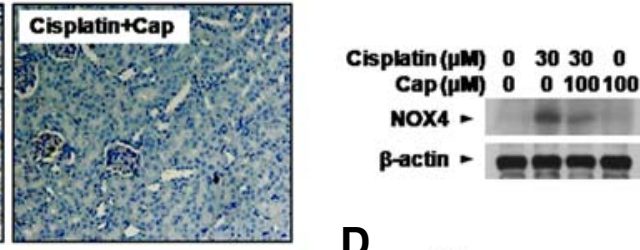

D

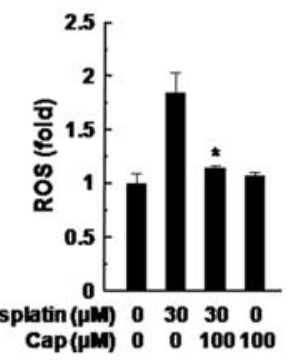

Fig. 3. Effect of capsaicin pretreatment on cisplatin-induced oxidative stress markers. Kidney sections were immunohistochemically stained with NOX4 (A) or 4-HNE (C) antibody. Cont, PBS (phosphate-buffered saline)-treated group; Cisplatin, cisplatin only treated group; Cisplatin + Cap, cisplatin and $10 \mathrm{mg} / \mathrm{kg}$ capsaicin combined group. (B, D) HK-2 cells were treated with 30 $\mu \mathrm{M}$ cisplatin for $24 \mathrm{~h}$ in the presence or absence of $100 \mu \mathrm{M}$ capsaicin. NOX4 expression was measured by Western blotting (B). To determine ROS level, cells were incubated with $10 \mu \mathrm{M} \mathrm{H} 2$ DCFDA at $37^{\circ} \mathrm{C}$ for $60 \mathrm{~min}$ and

then fluorescence intensity was recorded using a fluorometer (D). ${ }^{*} p<0.05$ by one-way ANOVA compared with the cisplatin $(n=3)$.

\section{Capsaicin attenuates cisplatin-induced oxidative stress in} mice

Reactive oxygen species (ROS), which induce oxidative stress, are important in the pathogenesis of cisplatin-induced renal injury, and $\mathrm{NAD}(\mathrm{P}) \mathrm{H}$ oxidase 4 (NOX4) is one of the major sources of ROS generation in cisplatin-treated kidneys (Mukhopadhyay et al., 2010; 2011). Elevated levels of intracellular ROS lead to the oxidation of several cellular molecules, including proteins and lipids, resulting in cellular stress (Chen et al.,
1995). IHC and Western blot analysis revealed that cisplatin treatment increased the expression of NOX4, whereas capsaicin pretreatment attenuated the expression of NOX4 in the kidney tissues and HK-2 cells (Figs. $3 \mathrm{~A}$ and $3 \mathrm{~B}$ ). Similar to the effect of capsaicin on the expression of NOX4, capsaicin pretreatment also markedly blocked the expression of 4-HNE, a biomarker for lipid peroxidation, in the kidney tissues and intracellular ROS level in HK-2 cells in response to cisplatin treatment (Figs. 3C and 3D). Taken together, these results suggest 
A

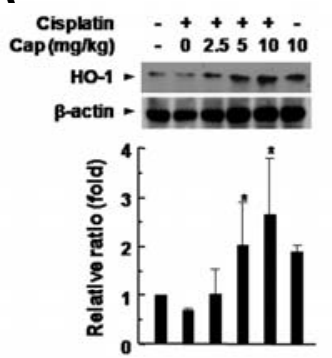

E

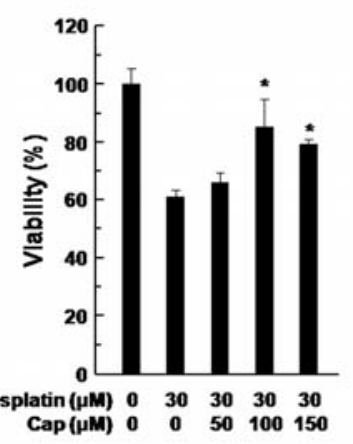

B

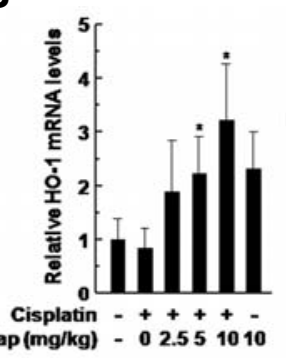

$F$

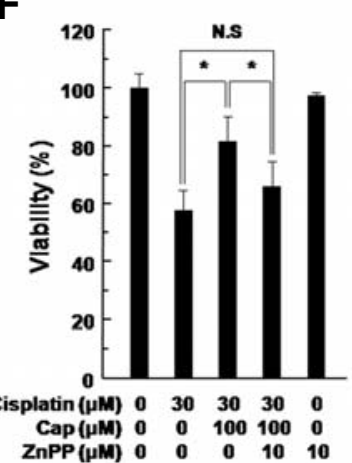

C

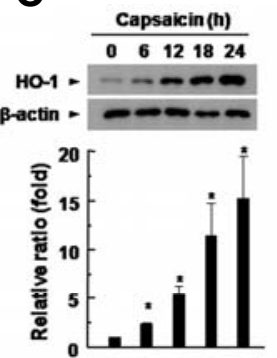

G

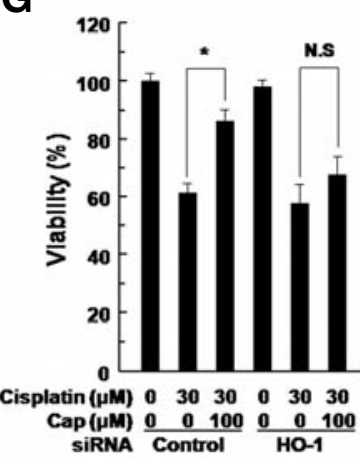

Fig. 4. Effect of capsaicin pretreatment on the expression of $\mathrm{HO}-1$ and cisplatin-induced cytotoxicity. (A) For the quantitative analysis of protein expression level, kidney lysates were analyzed by Western blotting for $\mathrm{HO}-1$ and Actin (upper panel) and signal intensities were quantified (lower panel). ${ }^{*} p<0.05$ by one-way ANOVA compared with the control $(n=3)$. (B) Real-time PCR analysis of $\mathrm{HO}-1$ expression in the kidney. Results were normalized to GAPDH expression and expressed as fold change compared with control. * $p$ $<0.05$ by one-way ANOVA compared with the control $(n=3)$. (C) Western blots and corresponding densitometric analyses of $\mathrm{HO}-1$ expression in HK-2 cells at the indicated times after capsaicin treatment $(100 \mu \mathrm{M})$. ${ }^{*} p<0.05$ by one-way ANOVA compared with the control $(n=3)$. (D) Real-time $\mathrm{PCR}$ analysis of $\mathrm{HO}-1$ expression

in HK-2 cells at the indicated times after capsaicin treatment $(100 \mu \mathrm{M})$. Results were normalized to GAPDH expression and expressed as fold change compared with control. * $P<0.05$ by one-way ANOVA compared with the control $(n=3)$. (E-G) Cell viability was determined by MTT assay. Cell viability was expressed as a percentage of controls. ${ }^{*} p<0.05$ by one-way ANOVA compared with the cisplatin $(n=3)$. N.S, not significant.

that capsaicin prevents cisplatin-induced renal injury by suppressing critical mediators of inflammation and ROS generation.

\section{Capsaicin attenuates cisplatin-cytotoxicity through \\ induction of $\mathrm{HO}-1$ expression}

Previous studies reported that capsaicin increased HO-1 expression through activation of Nrf2 signaling in HepG2 cells (Joung et al., 2007). In addition, we have previously demonstrated that increased $\mathrm{HO}-1$ expression leads to the protection of hearing function against cisplatin-induced hearing impairment (So et al., 2008). Thus, we investigated whether the protective effect of capsaicin is mediated by the induction of HO-1 expression in vivo and in vitro. Western blot and RT-PCR analysis showed that capsaicin dose-dependently increased the expression of $\mathrm{HO}-1$ protein (Fig. 4A) and mRNA (Fig. 4B) in the kidney and also time-dependently increased the expression of $\mathrm{HO}-1$ in vitro (Figs. 4C and 4D). To determine the role of $\mathrm{HO}-1$ in capsaicin-mediated protection against cisplatin cytotoxicity, HK-2 cells were treated with $30 \mu \mathrm{M}$ cisplatin and $100 \mu \mathrm{M}$ capsaicin in the presence and absence of $\mathrm{HO}$ inhibitor ZnPP IX or siRNA-HO-1. Capsaicin alone did not affect cell viability of HK-2 cells (data not shown). An MTT assay showed that pretreatment with capsaicin significantly increased the cell viability of cisplatin-treated HK-2 cells, while either treatment with $\mathrm{HO}$ inhibitor ZnPP IX or transfection of HO-1 specific siRNA abolished the cytoprotective effect of capsaicin (Figs. 4E-4G). This finding suggests that the induction of $\mathrm{HO}-1$ by capsaicin treatment may play a key role in the protection against cisplatinmediated renal toxicity.

\section{DISCUSSION}

In this study, we have demonstrated that capsaicin increases $\mathrm{HO}-1$ expression and ameliorates cisplatin-induced renal injury through the inhibition of oxidative stress and the inflammatory response. Cisplatin treatment increased the expression of NOX4 and 4-HNE in kidney tissues, but capsaisin pretreatment reduced the expression of these ROS-related proteins. In addition, capsaicin pretreatment markedly decreased the expression of inflammatory mediators, including TLR4, HMGB1, and AGEs. Furthermore, in vivo studies with mice and in vitro studies with HK-2 cells revealed that capsaicin treatment increased HO-1 expression, which reduced cisplatin-mediated cell death. Accordingly, pharmacological inhibition or knockdown of HO-1 effectively reversed the protective effect of capsaicin on cisplatin-induced cell death. These findings indicate that induction of HO-1 by capsaicin inhibits cisplatin-induced renal cytotoxicity.

Numerous studies suggest that inflammation is associated with cisplatin-induced nephrotoxicity (Kuhad et al., 2007; Mitazaki et al., 2013; Mukhopadhyay et al., 2010; 2011; Sahu et al., 2013). The inflammatory response to cisplatin is characterized by the infiltration of immune cells and upregulation of proinflammatory cytokines. Previously, we and others have demonstrated an important role for pro-inflammatory cytokines, including TNF- $\alpha$, in the pathogenesis of cisplatin-induced tissue injury, and the inhibition of TNF- $\alpha$ production or function markedly abolished cisplatin-induced ototoxicity and nephrotoxicity (Ramesh and Reeves, 2002; So et al., 2007). Toll-like receptors (TLRs), which regulate innate and adaptive immune responses during tissue injury by recognizing pathogen-associated mole- 
cular patterns as well as damage-associated molecular patterns, also mediate inflammatory responses. In the kidney, tubular epithelial cells express various TLRs including TLR2, TLR4, and TLR6, suggesting that these TLRs might contribute to the inflammatory responses in renal injury. More specifically, TLR4 is studied most extensively in cisplatin-induced nephrotoxicity. Previous studies demonstrated that cisplatin-induced acute renal failure is dependent on TLR4 signaling. Cenedeze et al. (2007) reported that protection against cisplatin-induced renal injury in mice could be conferred by the expression of TLR4 receptor with a point. In addition, increased production of proinflammatory cytokines and chemokines in response to cisplatin was reduced in TLR4 deficient mice (Zhang et al., 2008). These observations indicate that TLR4 activation is a key upstream event leading to cytokine production, inflammation, and renal injury upon cisplatin treatment. However, it remains unclear whether traditional TLR4 ligands like HMGB1 and AGEs are also involved in cisplatin-induced nephrotoxicity. In this study, we showed that capsaicin downregulates the expression of inflammatory mediators in kidney, consistent with the report that capsaicin treatment can attenuate systemic inflammatory cytokines during sepsis in rats. Moreover, we demonstrated for the first time that cisplatin-induced renal injury is associated with the increased expression of TLR4 and its ligands, HMGB1, and AGEs. Interestingly, we found differential expression of HMGB1 and AGEs in cisplatin-treated kidneys. The expression of HMGB1 mainly increased in the glomeruli cells, while AGEs were mainly expressed in the tubular cells. Consistent with this finding, HMGB1 was recently shown to attract macrophages by inducing MCP-1 release from renal tubules, which promotes granulomatous inflammation and worsens nephropathy, including tubular damage and glomerular injury (Oyama et al., 2010).

Several studies also suggest that cisplatin-induced nephrotoxicity is largely mediated by lipid peroxidation and ROS generation. In fact, various antioxidants were shown to be effective in protection against CDDP-induced nephrotoxicity (Pabla and Dong, 2008). In particular, capsaicin and/or its analogues were suggested to have antioxidant activities and thus be potentially protective against cisplatin-mediated renal injury in rats (Shimeda et al., 2005). However, the mechanisms underlying the antioxidant and protective effects of capsaicin were poorly understood. In this study, we attempted to investigate the possible molecular mechanisms underlying the cytoprotective effects of capsaicin with prime focus on the upregulation of HO-1. HO-1 is an enzyme with potent antioxidant activity, and upregulation of $\mathrm{HO}-1$ expression is recognized as a key event to maintain cellular antioxidant capacity. Several studies have demonstrated that HO-1 mRNA is induced in the kidney as an early defense mechanism in various nephrotoxic animal models, such as ischemia/reperfusion and nephrotoxin-induced acute kidney injury (Agarwal et al., 1995; Nath et al., 2000). Genetic depletion or chemical inhibition of HO-1 significantly worsens kidney function and tubular injury in response to cisplatin treatment, further supporting a protective role for $\mathrm{HO}-1$ expression in cisplatin-induced renal injury (Agarwal et al., 1995; Shiraishi et al., 2000). Furthermore, we and others have documented that HO1 activation exerts potent anti-inflammatory functions in a various pathophysiological conditions, including cisplatin-induced ototoxicity and hepatic ischemia/reperfusion injury (Sass et al., 2012; So et al., 2008). We demonstrated in this report that capsaicin increased the expression of $\mathrm{HO}-1$ in a dose- and timedependent manner without causing severe toxicity in kidney tissues or HK-2 cells. Moreover, specific inhibition of HO-1 function or expression abolished the protective effect of capsaicin against cisplatin-induced cytotoxicity in HK-2 cells. These results indicate that $\mathrm{HO}-1$ is one of the essential regulators for the protective effects of capsaicin on cisplatin-induced nephrotoxicity. Although $\mathrm{HO}-1$ could be induced through various transcriptional activations, such as AP-1, STAT and Nrf2, numerous studies have reported that $\mathrm{HO}-1$ induction is mainly regulated by Nrf2. Thus, we also considered the possibility that other Nrf2-regulated genes may contribute the protective effects of capsaicin against cisplatin. To address this question, we iexamined the mRNA expression profiles of various Nrf2-regulated genes, including NQO1, GCLC, GCLM, and GSS. In contrast to expression of HO-1, mRNA expressions of these genes were not affected by capsaicin in the kidney tissues (data not shown). Therefore, it remains unclear which gene(s) cooperate with $\mathrm{HO}$ 1 that could confer the protective effects of capsaicin against cisplatin-induced nephrotoxicity.

In conclusion, we showed that capsaicin ameliorates cisplatin-induced nephrotoxicity by effectively suppressing oxidative damages and inflammatory responses, and that $\mathrm{HO}-1$ induction is central to the protective mechanism of capsaicin. In the present study, we suggest that the activation of $\mathrm{HO}-1$ by the regular intake of natural phytochemicals may represent one of the most practical and feasible ways to reduce the risk of cisplatininduced toxicity.

\section{ACKNOWLEDGMENTS}

This work was supported by two National Research Foundation of Korea (NRF) grants funded by the Korean government [Ministry of Education, Science, and Technology (MEST)]: (No. 2011-0028866) and (No. 2011-0030715).

\section{REFERENCES}

Agarwal, A., Balla, J., Alam, J., Croatt, A.J., and Nath, K.A. (1995). Induction of heme oxygenase in toxic renal injury: a protective role in cisplatin nephrotoxicity in the rat. Kidney Int. 48, 1298-1307.

Arany, I., and Safirstein, R.L. (2003). Cisplatin nephrotoxicity. Semin. Nephrol. 23, 460-464.

Caterina, M.J., Schumacher, M.A., Tominaga, M., Rosen, T.A., Levine, J.D., and Julius, D. (1997). The capsaicin receptor: a heat-activated ion channel in the pain pathway. Nature 389, 816-824

Cenedeze, M.A., Goncalves, G.M., Feitoza, C.Q., Wang, P.M., Damiao, M.J., Bertocchi, A.P., Pacheco-Silva, A., and Camara, N.O. (2007). The role of toll-like receptor 4 in cisplatin-induced renal injury. Transplant Proc. 39, 409-411.

Chen, Q., Fischer, A., Reagan, J.D., Yan, L.J., and Ames, B.N. (1995). Oxidative DNA damage and senescence of human diploid fibroblast cells. Proc. Natl. Acad. Sci. USA 92, 4337-4341.

Cohen, S.M., and Lippard, S.J. (2001). Cisplatin: from DNA damage to cancer chemotherapy. Prog. Nucleic Acid Res. Mol. Biol. 67, 93-130.

Hartsfield, C.L., Alam, J., and Choi, A.M. (1999). Differential signaling pathways of $\mathrm{HO}^{-1}$ gene expression in pulmonary and systemic vascular cells. Am. J. Physiol. 277, L1133-1141.

Huang, S.P., Chen, J.C., Wu, C.C., Chen, C.T., Tang, N.Y., Ho, Y.T., Lo, C., Lin, J.P., Chung, J.G., and Lin, J.G. (2009). Capsaicin-induced apoptosis in human hepatoma HepG2 cells. Anticancer Res. 29, 165-174.

Joe, B., and Lokesh, B.R. (1994). Role of capsaicin, curcumin and dietary $n-3$ fatty acids in lowering the generation of reactive oxygen species in rat peritoneal macrophages. Biochim. Biophys. Acta 1224, 255-263.

Joung, E.J., Li, M.H., Lee, H.G., Somparn, N., Jung, Y.S., Na, H.K., Kim, S.H., Cha, Y.N., and Surh, Y.J. (2007). Capsaicin induces heme oxygenase- 1 expression in HepG2 cells via activation of PI3K-Nrf2 signaling: NAD $(P) H$ :quinone oxidoreductase as a potential target. Antioxid. Redox Signal. 9, 2087-2098.

Kogure, K., Goto, S., Nishimura, M., Yasumoto, M., Abe, K., Ohiwa, C., Sassa, H., Kusumi, T., and Terada, H. (2002). Mechanism of 
potent antiperoxidative effect of capsaicin. Biochim. Biophys. Acta 1573, 84-92.

Kuhad, A., Pilkhwal, S., Sharma, S., Tirkey, N., and Chopra, K. (2007). Effect of curcumin on inflammation and oxidative stress in cisplatin-induced experimental nephrotoxicity. J. Agric. Food Chem. 55, 10150-10155.

Kuriakose, G.C., and Kurup, M.G. (2008). Evaluation of renoprotective effect of Aphanizomenon flos-aquae on cisplatin-induced renal dysfunction in rats. Ren. Fail. 30, 717-725.

Lee, P.J., Camhi, S.L., Chin, B.Y., Alam, J., and Choi, A.M. (2000). AP-1 and STAT mediate hyperoxia-induced gene transcription of heme oxygenase-1. Am. J. Physiol. Lung Cell Mol. Physiol. 279, L175-182.

Luke, D.R., Vadiei, K., and Lopez-Berestein, G. (1992). Role of vascular congestion in cisplatin-induced acute renal failure in the rat. Nephrol. Dial. Transplant. 7, 1-7.

Maheshwari, R.A., Sailor, G.U., Patel, L., and Balaraman, R. (2013) Amelioration of cisplatin-induced nephrotoxicity by statins. Indian J. Pharmacol. 45, 354-358.

Maines, M.D. (1988). Heme oxygenase: function, multiplicity, regulatory mechanisms, and clinical applications. FASEB. J. 2, 25572568.

Mitazaki, S., Hashimoto, M., Matsuhashi, Y., Honma, S., Suto, M., Kato, N., Nakagawasai, O., Tan-No, K., Hiraiwa, K., Yoshida, M., et al. (2013). Interleukin-6 modulates oxidative stress produced during the development of cisplatin nephrotoxicity. Life Sci. 92, 694-700.

Mukhopadhyay, P., Rajesh, M., Pan, H., Patel, V., Mukhopadhyay, B., Batkai, S., Gao, B., Hasko, G., and Pacher, P. (2010). Cannabinoid-2 receptor limits inflammation, oxidative/nitrosative stress, and cell death in nephropathy. Free Radic. Biol. Med. 48, 457-467.

Mukhopadhyay, P., Horvath, B., Kechrid, M., Tanchian, G., Rajesh, M., Naura, A.S., Boulares, A.H., and Pacher, P. (2011). Poly(ADPribose) polymerase-1 is a key mediator of cisplatin-induced kidney inflammation and injury. Free Radic. Biol. Med. 51, 17741788.

Nath, K.A., Haggard, J.J., Croatt, A.J., Grande, J.P., Poss, K.D., and Alam, J. (2000). The indispensability of heme oxygenase-1 in protecting against acute heme protein-induced toxicity in vivo. Am. J. Pathol. 156, 1527-1535.

Okada, Y., and Okajima, H. (2001). Antioxidant effect of capsaicin on lipid peroxidation in homogeneous solution, micelle dispersions and liposomal membranes. Redox Rep. 6, 117-122.

Owuor, E.D., and Kong, A.N. (2002). Antioxidants and oxidants regulated signal transduction pathways. Biochem. Pharmacol. $64,765-770$.
Oyama, Y., Hashiguchi, T., Taniguchi, N., Tancharoen, S., Uchimura, T., Biswas, K.K., Kawahara, K., Nitanda, T., Umekita, Y., Lotz, M., et al. (2010). High-mobility group box-1 protein promotes granulomatous nephritis in adenine-induced nephropathy. Lab. Invest. 90, 853-866.

Pabla, N., and Dong, Z. (2008). Cisplatin nephrotoxicity: mechanisms and renoprotective strategies. Kidney Int. 73, 994-1007.

Ramesh, G., and Reeves, W.B. (2002). TNF-alpha mediates chemokine and cytokine expression and renal injury in cisplatin nephrotoxicity. J. Clin. Invest. 110, 835-842.

Sahu, B.D., Kuncha, M., Sindhura, G.J., and Sistla, R. (2013). Hesperidin attenuates cisplatin-induced acute renal injury by decreasing oxidative stress, inflammation and DNA damage. Phytomedicine 20, 453-460.

Sass, G., Barikbin, R., and Tiegs, G. (2012). The multiple functions of heme oxygenase-1 in the liver. Z. Gastroenterol. 50, 34-40.

Shimeda, Y., Hirotani, Y., Akimoto, Y., Shindou, K., ljiri, Y., Nishihori, T., and Tanaka, K. (2005). Protective effects of capsaicin against cisplatin-induced nephrotoxicity in rats. Biol. Pharm. Bull. 28, 1635-1638

Shiraishi, F., Curtis, L.M., Truong, L., Poss, K., Visner, G.A., Madsen, K., Nick, H.S., and Agarwal, A. (2000). Heme oxygenase-1 gene ablation or expression modulates cisplatin-induced renal tubular apoptosis. Am. J. Physiol. Renal Physiol. 278, F726-736.

Siddik, Z.H. (2003). Cisplatin: mode of cytotoxic action and molecular basis of resistance. Oncogene 22, 7265-7279.

So, H., Kim, H., Lee, J.H., Park, C., Kim, Y., Kim, E., Kim, J.K., Yun, K.J., Lee, K.M., Lee, H.Y., et al. (2007). Cisplatin cytotoxicity of auditory cells requires secretions of proinflammatory cytokines via activation of ERK and NF-kappaB. J. Assoc. Res. Otolaryngol. 8, 338-355.

So, H., Kim, H., Kim, Y., Kim, E., Pae, H.O., Chung, H.T., Kim, H.J., Kwon, K.B., Lee, K.M., Lee, H.Y., et al. (2008). Evidence that cisplatin-induced auditory damage is attenuated by downregulation of pro-inflammatory cytokines via Nrf2/HO-1. J. Assoc. Res. Otolaryngol. 9, 290-306.

Szallasi, A., and Blumberg, P.M. (1999). Vanilloid (Capsaicin) receptors and mechanisms. Pharmacol. Rev. 51, 159-212.

Wang, D., and Lippard, S.J. (2005). Cellular processing of platinum anticancer drugs. Nat. Rev. Drug Discov. 4, 307-320.

Zhang, J., Nagasaki, M., Tanaka, Y., and Morikawa, S. (2003). Capsaicin inhibits growth of adult T-cell leukemia cells. Leuk. Res. 27, 275-283.

Zhang, B., Ramesh, G., Uematsu, S., Akira, S., and Reeves, W.B. (2008). TLR4 signaling mediates inflammation and tissue injury in nephrotoxicity. J. Am. Soc. Nephrol. 19, 923-932. 